\title{
Viologen-Decorated TEMPO for Neutral Aqueous Organic Redox Flow Batteries
}

\author{
Shuzhi Hu, ${ }^{1,2}$ Liwen Wang, Xianzhi Yuan, ${ }^{1}$ Zhipeng Xiang, ${ }^{1}$ Mingbao Huang, ${ }^{1}$ Peng Luo, ${ }^{1}$ \\ Yufeng Liu, ${ }^{1}$ Zhiyong Fu, ${ }^{1}$ and Zhenxing Liang ${ }^{1}$ \\ ${ }^{1}$ Key Laboratory of Fuel Cell Technology of Guangdong Province, School of Chemistry and Chemical Engineering, South China \\ University of Technology, Guangzhou 510641, China \\ ${ }^{2}$ School of Materials Science and Engineering, Sun Yat-sen University, Guangzhou 510275, China
}

Correspondence should be addressed to Zhiyong Fu; zyfu@scut.edu.cn and Zhenxing Liang; zliang@scut.edu.cn

Received 23 June 2021; Accepted 26 July 2021; Published 14 August 2021

Copyright (C 2021 Shuzhi Hu et al. Exclusive Licensee Beijing Institute of Technology Press. Distributed under a Creative Commons Attribution License (CC BY 4.0).

A novel electroactive organic molecule, viz., 1-(1-oxyl-2,2,6,6-tetramethylpiperidin-4-yl)-1' -(3-(trimethylammonio)propyl)4,4'-bipyridinium trichloride ((TPABPy) $\left.\mathrm{Cl}_{3}\right)$, is synthesized by decorating 2,2,6,6-tetramethylpiperidin-1-oxyl (TEMPO) with viologen, which is used as the positive electrolyte in neutral aqueous redox flow battery (ARFB). Extensive characterizations are performed to investigate the composition/structure and the electrochemical behavior, revealing the favorable effect of introducing the cationic viologen group on the electroactive TEMPO. Salient findings are as follows. First, the redox potential is elevated from $+0.745 \mathrm{~V}$ for TEMPO to $+0.967 \mathrm{~V}$ for decorated TEMPO, favoring its use as the positive electrolyte. Such an elevation originates from the electron-withdrawing effect of the viologen unit, as evidenced by the nuclear magnetic resonance and single crystal structure analysis. Second, linear sweep voltammetry reveals that the diffusion coefficient is $2.97 \times 10^{-6} \mathrm{~cm}^{2} \mathrm{~s}^{-1}$, and the rate constant of the one-electron transfer process is $7.50 \times 10^{-2} \mathrm{~cm} \mathrm{~s}^{-1}$. The two values are sufficiently high as to ensure low concentration and kinetics polarization losses during the battery operation. Third, the permeability through anion-exchange membrane is as low as $1.80 \times 10^{-11} \mathrm{~cm}^{2} \mathrm{~s}^{-1}$. It is understandable as the positive-charged viologen unit prevents the molecule from permeating through the anion exchange membrane by the Donnan effect. Fourth, the ionic nature features a decent conductivity and thus eliminates the use of additional supporting electrolyte. Finally, a flow battery is operated with $1.50 \mathrm{M}$ (TPABPy) $\mathrm{Cl}_{3}$ as the positive electrolyte, which affords a high energy density of $19.0 \mathrm{Wh} \mathrm{L}^{-1}$ and a stable cycling performance with capacity retention of $99.98 \%$ per cycle.

\section{Introduction}

Wide application of clean energy (e.g., solar and wind energy) in near future needs urgent development of electrochemical energy storage technologies [1]. Redox flow battery (ARFB) is one of the most important large-scale technologies, and all-vanadium redox flow battery (VRFB) has proved itself as one option [2]. The current challenge is the high cost of the electrolyte due to the resource scarcity of vanadium. Considerable efforts have been then devoted to developing alternative electrolytes [3]. Electroactive organic merit of element abundance, low cost, and flexible molecular control over the electrochemical features, which have been regarded as key to develop next-generation RFBs [4].
2,2,6,6-Tetramethylpiperidin-1-oxyl (TEMPO) shows reversible one-electron storage and high redox potential in organic solvent, which has been applied as the positive electrolyte in nonaqueous RFB since 2011 [5]. Hydrophilic functionalization of the molecule makes it possible be used in an aqueous redox flow battery (ARFB). For example, Liu found that the hydroxyl-functionalized TEMPO 4-hydroxy-2,2,6,6tetramethylpiperidin-1-oxyl (4-HO-TEMPO) showed a high aqueous solubility of $2.1 \mathrm{M}$ [6]. Then, they developed an ARFB by using $0.5 \mathrm{M}$ methyl viologen(-)/4-HO-TEMPO(+) for demonstration. Quaternization has been proved as an effective strategy to increase the solubility and redox potential. Schubert developed N,N,N-2,2,6,6-heptamethylpiperidinyl oxy-4-ammonium chloride (TEMPTMA) with a high 


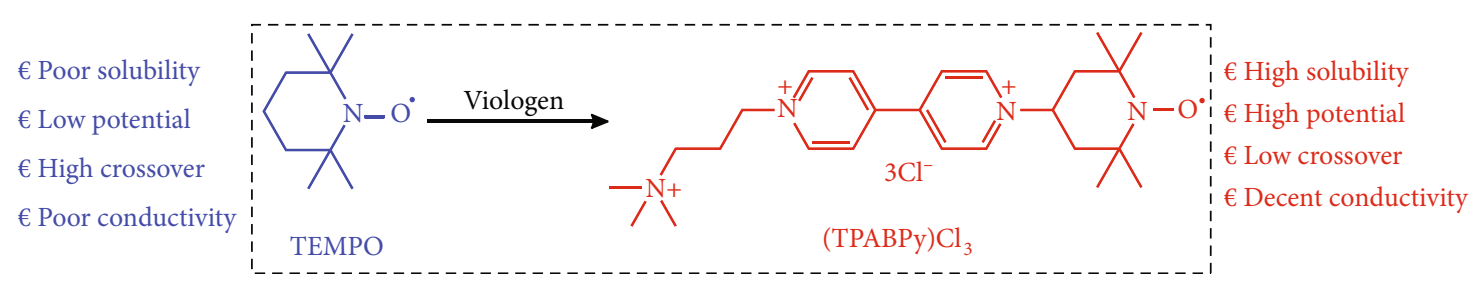

Scheme 1: Decoration of TEMPO.

redox potential of $0.99 \mathrm{~V}$ and yielded a high energy density of 25.2 $\mathrm{Wh} \mathrm{L}^{-1}$ in an ARFB [7]. It is argued that its high potential might be electrochemically unstable in aqueous solution [8]. Yang synthesized a more stable molecule, viz., 4-[3-(trimethylammonio)propoxy]-2,2,6,6-tetramethylpiperidine-1oxyl chloride (TMAP-TEMPO), which showed a potential of $0.81 \mathrm{~V}$ and an energy density of $12.0 \mathrm{Wh} \mathrm{L}^{-1}$ in an ARFB [9]. Besides, the same author claimed that the neutral TEMPO molecule may easily penetrate the separator membrane from the positive to negative side, which threatens the battery stability during the operation. The quaternary ammonium cation can effectively prevent the molecule against its crossover through the anion exchange membrane (AEM) by the Donnan effect and thus improve the battery stability. Literature survey reveals that the practical application of TEMPO as the positive electrolyte needs more work on molecule engineering to increase the aqueous solubility, elevate the redox potential, and prevent its crossover.

In this work, we present a viologen-functionalized TEMPO compound, viz., 1-(1-oxyl-2,2,6,6-tetramethylpiperidin-4-yl)-1' -(3-(trimethylammonio)propyl)-4,4' -bipyridinium trichloride (TPABPy) $\mathrm{Cl}_{3}$ (Scheme 1). The rational design of this TEMPO derivative is as follows. First, viologen is highly water-soluble and thus promotes the aqueous solubility. Second, the strong electron withdrawing effect of viologen is able to elevate its redox potential. Third, the incorporation of densely positive-charge unit can minimize its crossover through AEMs. Fourth, the salt nature of viologen endows TEMPO with a decent conductivity in an aqueous solution. Experiment result confirms that the membrane (Selemion, AMV) permeability of (TPABPy) $\mathrm{Cl}_{3}$ is reduced to be as low as $1.80 \times 10^{-11} \mathrm{~cm}^{2} \mathrm{~s}^{-1}$. When paired with bis(3-trimethylammonio)propyl viologen tetrachloride (BTMAP-Vi), an ARFB using $1.50 \mathrm{M}$ (TPABPy) $\mathrm{Cl}_{3}$ without adding external electrolyte delivers a high energy density up to $19.0 \mathrm{Wh} \mathrm{L}^{-1}$ and a stable cycling performance with capacity retention of $99.98 \%$ per cycle.

\section{Materials and Methods}

2.1. Materials. All of the reagents were commercially available. 4,4' ${ }^{\prime}$-Bipyridine (98\%, Shanghai Aladdin Bio-Chem Technology Co., Ltd., China), 1-chloro-2,4-dinitrobenzene (98\%, Adamas Reagent Co., Ltd., Shanghai, China), 4-amino-2,2,6,6tetramethylpiperidine-1-oxyl (97\%, Shanghai Aladdin BioChem Technology Co., Ltd., China), N-(3-bromopropyl)N,N,N-trimethylammonium bromide (BPTMABr, 97\%, Ark Pharm, Inc., USA), potassium hexafluorophosphate $\left(\mathrm{KPF}_{6}\right.$, 99\%, Shanghai Aladdin Bio-Chem Technology Co., Ltd.,
China), tetrabutylammonium chloride (TBAC, 99\%, Adamas Reagent Co., Ltd., Shanghai, China), $\mathrm{KCl}$ (analytical pure, Guangzhou Chemical Reagent Factory, China), dimethyl formamide (DMF, analytical pure, Chinasun Specialty Products Co., Ltd., China), acetonitrile (analytical pure, Shanghai Lingfeng Chemical Reagent Co., Ltd., China), ethanol (analytical pure, Sinopharm Chemical Reagent Co., Ltd., China), and acetone (analytical pure, Guangzhou Chemical Reagent Factory, China) were used without further purification.

2.2. Synthesis of (TPABPy)Cl 3 . The synthetic route of (TPAB$\mathrm{Py}) \mathrm{Cl}_{3}$ is shown in Scheme 1. In the first step, a mixture consisting of acetone $(250 \mathrm{~mL}), 1$-chloro-2,4-dinitrobenzene $(12.150 \mathrm{~g}, \quad 60.0 \mathrm{mmol})$ and $4,4^{\prime}$-bipyridine $\quad(9.360 \mathrm{~g}$, $60.0 \mathrm{mmol}$ ) was stirred at $75^{\circ} \mathrm{C}$ for $12 \mathrm{~h}$. The resultant light grey precipitate was washed by acetone $(250 \mathrm{~mL})$, filtered, and finally, dried under vacuum at $40^{\circ} \mathrm{C}$ to get (DPPy)Cl (1(2,4-dinitrophenyl)-4-(4-pyridyl)pyridinium chloride). In the second step, (DPPy)Cl (14.340 g, $40.0 \mathrm{mmol})$ and 4-amino2,2,6,6-tetramethylpiperidin-1-oxyl (8.208g, $48.0 \mathrm{mmol})$ were dissolved in ethanol-water solution $(250 \mathrm{~mL})$ with the volumetric ratio of $4: 1$. The reactant was stirred at $90^{\circ} \mathrm{C}$ for $12 \mathrm{~h}$ and then cooled to room temperature, and the orange-red filtrate was collected by vacuum filtration. The solvent was then removed by vacuum rotary evaporation. Acetone $(150 \mathrm{~mL})$ was added in and refluxed at $70^{\circ} \mathrm{C}$ for $6 \mathrm{~h}$. The orange-red product was filtered and washed with acetone $(100 \mathrm{~mL})$ to get 1-(1-oxyl-2,2,6,6-tetramethylpiperidin-4-yl)-4-(4-pyridyl)pyridinium chloride $((\mathrm{TPPy}) \mathrm{Cl})$. In the third step, (TPPy)Cl (6.930 g, $20.0 \mathrm{mmol}$ ) and $\mathrm{N}$-(3-bromopropyl)$\mathrm{N}, \mathrm{N}, \mathrm{N}$-trimethylammonium bromide $(7.830 \mathrm{~g}, 30.0 \mathrm{mmol})$ were dispersed in anhydrous DMF $(60 \mathrm{~mL})$ and stirred at $100^{\circ} \mathrm{C}$ for $12 \mathrm{~h}$. Then, acetonitrile $(200 \mathrm{~mL})$ was added, and the solution was stirred at $25^{\circ} \mathrm{C}$ for $3 \mathrm{~h}$. The resultant yellow precipitate was filtered and washed with acetone $(100 \mathrm{~mL})$ to obtain (TPABPy) $\mathrm{Br}_{2} \mathrm{Cl}(10.5 \mathrm{~g})$. In the fourth step, (TPAB$\mathrm{Py}) \mathrm{Br}_{2} \mathrm{Cl}$ was dissolved in water, and (TPABPy) $\left(\mathrm{PF}_{6}\right)_{3}$ precipitate was obtained by adding excess $\mathrm{KPF}_{6}(14.3 \mathrm{~g})$. (TPABPy) $\left(\mathrm{PF}_{6}\right)_{3}$ was dissolved in acetonitrile $(250 \mathrm{~mL})$, in which TBAC (21.6g) was added to precipitate (TPABPy) $\mathrm{Cl}_{3}$. The product $(7.9 \mathrm{~g})$ was finally obtained by washing with acetonitrile $(250 \mathrm{~mL})$ and dried under vacuum at $40^{\circ} \mathrm{C}$.

2.3. Material Characterization. Crystal data of (TPABPy) $\mathrm{PF}_{6}$ was collected by a SuperNova diffractometer with Atlas S2 detector using a MoK $\alpha$ microfocus source. ${ }^{1} \mathrm{H}$ NMR spectra were obtained on an AVANCE III HD $500 \mathrm{MHz}$ (Bruker, Germany). UV-vis spectra were measured by a Shimadzu UV-2600 spectrometer. 


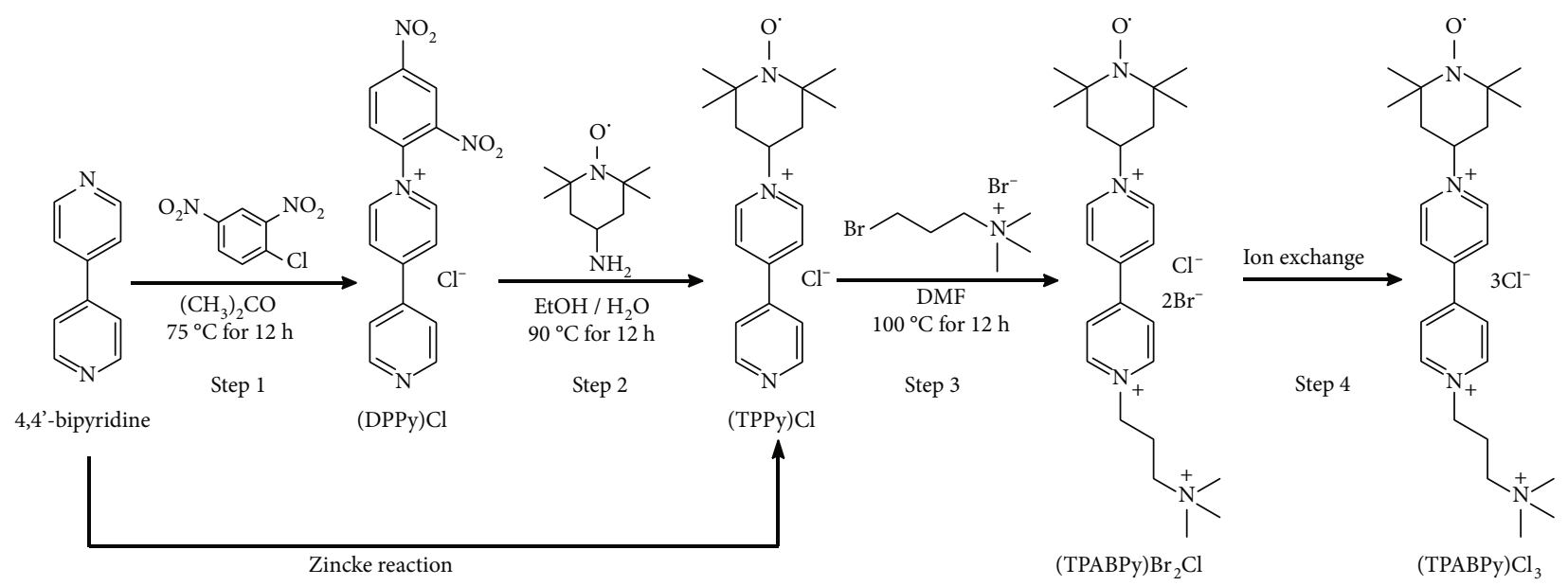

SCHEME 2: Synthesis protocol of (TPABPy) $\mathrm{Cl}_{3}$.

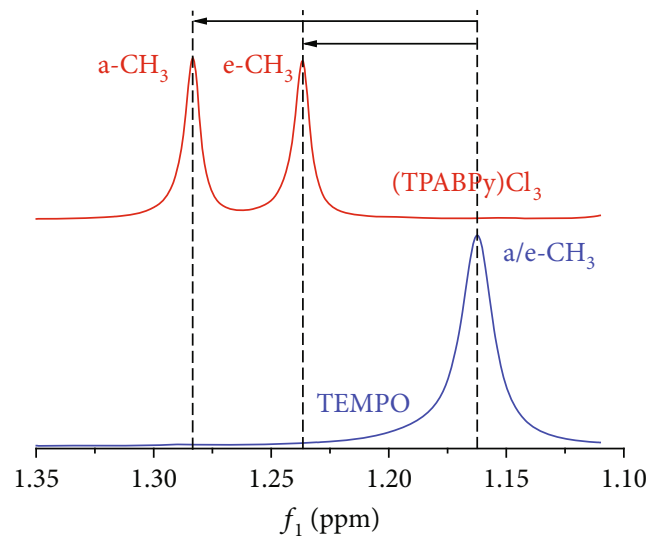

(a)

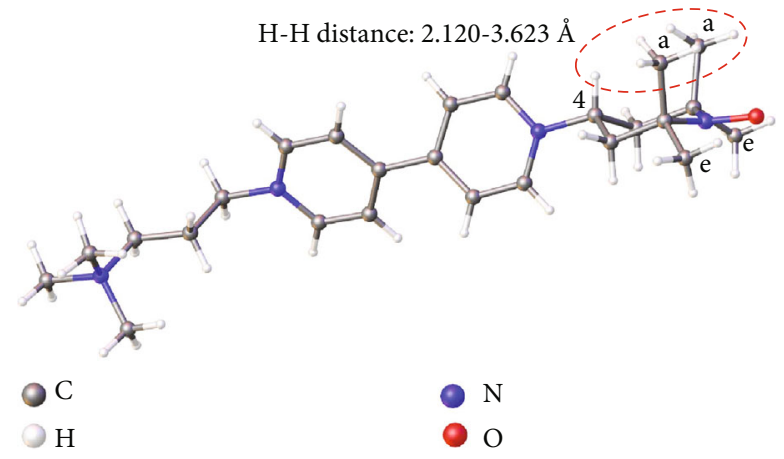

(b)

Figure 1: (a) Chemical shift of the methyl protons in the TEMPO ring; (b) the single crystal structure of (TPABPy) $)^{3+}$.

2.4. Permeability Measurements. A two-compartment $\mathrm{H}$-cell was used to evaluate the permeability of (TPABPy) $\mathrm{Cl}_{3}$ crossing the AMV anion exchange membrane. 0.10 M (TPAB$\mathrm{Py}) \mathrm{Cl}_{3}$ dissolved in $2.0 \mathrm{M} \mathrm{KCl}$ solution $(50 \mathrm{~mL})$ was filled into the donating side, and $2.0 \mathrm{M} \mathrm{KCl}$ solution $(50 \mathrm{~mL})$ was filled into the receiving side under continuously stirring. $2 \mathrm{~mL}$ of the solution was taken from the receiving side and tested by UV-vis spectrophotometry at different time intervals. The concentration was quantified from the established calibration curve. The permeability was calculated by the following equation:

$$
P=\frac{C_{t} V_{0} l}{A t\left(C_{0}-C_{t}\right)} \approx \frac{C_{t} V_{0} l}{A t C_{0}}
$$

where $P$ is the permeability $\left(\mathrm{cm}^{2} \mathrm{~s}^{-1}\right), C_{t}$ is the concentration $\left(\mathrm{mol} \mathrm{L}^{-1}\right)$ of active species at time $t$ in the receiving side, $V_{0}$ is the volume of the solution in either compartment, $A$ is the effective membrane area $\left(0.785 \mathrm{~cm}^{2}\right), l$ is the thickness of the membrane $(125 \mu \mathrm{m}), t$ is the the elapsed time $(\mathrm{s})$, and $C_{0}$ is the concentration of electrolyte in the donating compartment $\left(0.10 \mathrm{~mol} \mathrm{~L}^{-1}\right)$.
2.5. Electrochemical Measurements. Linear sweep voltammetry and cyclic voltammetry were carried out on a CHI $630 \mathrm{E}$ electrochemical workstation. The cyclic voltammograms (CVs) were collected at a scan rate of $100.0 \mathrm{mV} \mathrm{s}^{-1}$ on a standard three-electrode system (the working electrode: a glassy carbon disk (diameter: $3 \mathrm{~mm}$ ); the reference electrode: an $\mathrm{Ag} / \mathrm{AgCl}$ electrode; the counterelectrode: a platinum wire). Linear sweep voltammograms (LSVs) were collected by a rotating disk electrode (RDE, diameter: $5 \mathrm{~mm}$ ) at a scan rate of $5.0 \mathrm{mV} \mathrm{s}^{-1}$. Koutecky-Levich equation was used to determine the diffusion coefficient and the kinetic parameters:

$$
\frac{1}{i}=\frac{1}{i_{k}}+\frac{1}{0.62 n F A D^{2 / 3} v^{-1 / 6} C_{0}} \times \omega^{-1 / 2},
$$

where $i$ is the current (A) at a specific overpotential, $i_{k}$ is the kinetic current (A), $\omega$ is the rotating rate $\left(\operatorname{rad~s}^{-1}\right), C_{0}$ is the bulk concentration of (TPABPy) $\mathrm{Cl}_{3}(=1.0 \mathrm{mM}), v$ is the kinematic viscosity of $0.50 \mathrm{M}$ aqueous $\mathrm{KCl}$ solution $\left(=0.0088 \mathrm{~cm}^{2} \mathrm{~s}^{-1}\right), D$ is the diffusion coefficient $\left(\mathrm{cm}^{2} \mathrm{~s}^{-1}\right), A$ is the electrode $\left(=0.1963 \mathrm{~cm}^{2}\right), F$ is the Faraday constant $\left(=96485 \mathrm{C} \mathrm{mol}^{-1}\right)$, and $n$ is the electron transfer number $(=1)$. 


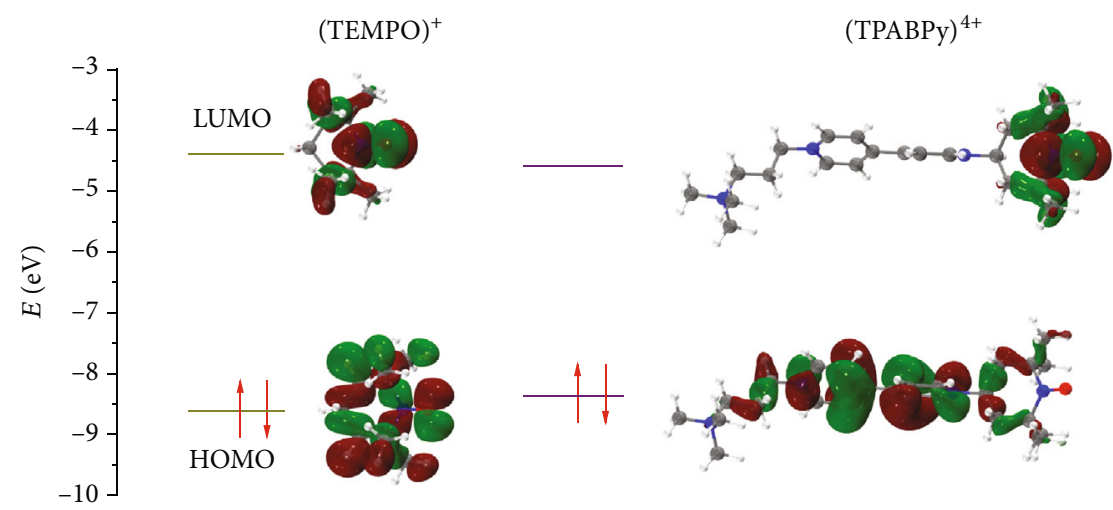

FIGURE 2: Frontier molecular orbitals and the relative energy levels of (TEMPO) ${ }^{+}$and (TPABPy) ${ }^{4+}$.

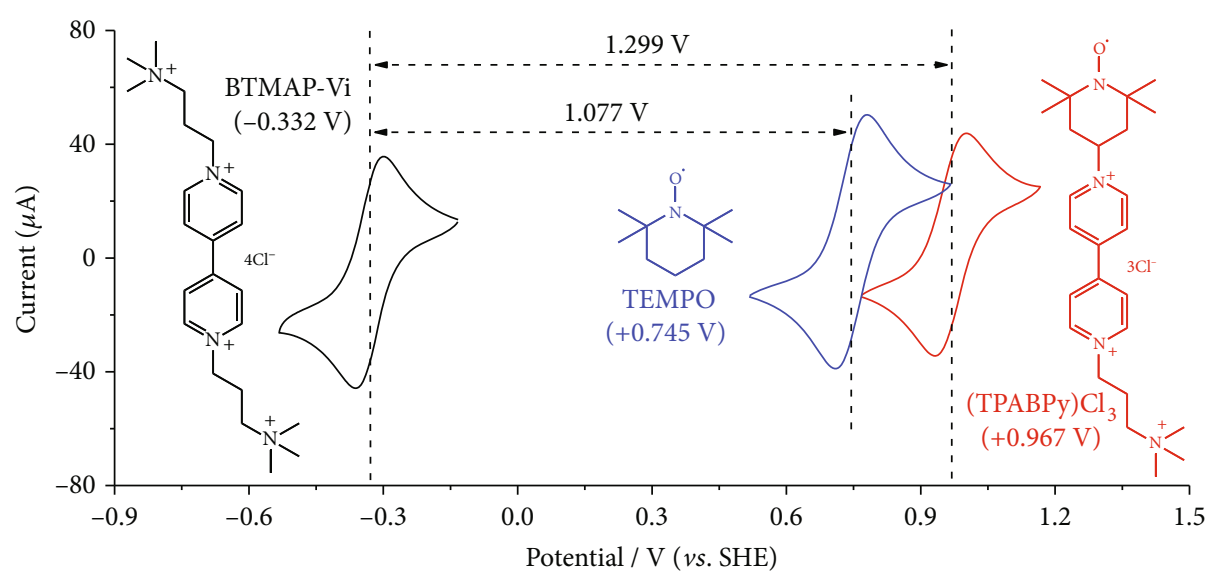

Figure 3: CVs of $4.0 \times 10^{-3} \mathrm{M}$ BTMAP-Vi, TEMPO, and (TPABPy) $\mathrm{Cl}_{3}$ in $0.50 \mathrm{M} \mathrm{KCl}$ solution at a scan rate of $100 \mathrm{mV} \mathrm{s}^{-1}$.

2.6. Flow Battery Test. A laboratory-scale flow battery was assembled as follows. Graphite felt electrodes $(3 \mathrm{~cm} \times 3 \mathrm{~cm}$ in size, $4 \mathrm{~mm}$ thick) were placed beside the titanium current collectors, and a Selemion AMV anion-exchange membrane was used as the separator. A peristaltic pump (Longer BT100-2 J) was used to circulate the electrolytes through the cell at a flow rate of $60.0 \mathrm{~mL} \mathrm{~min}^{-1}$. A Neware BTS 3000 battery testing system was used to evaluate the battery at $25^{\circ} \mathrm{C}$ in a 99.999\% argon-filled glove box (Universal 2440/750/900). Cycling experiment was performed by galvanostatic cycling with 1.5 and $0.5 \mathrm{~V}$ cut offs.

The battery performance was evaluated by two concentrations of (TPABPy) $\mathrm{Cl}_{3}$. For the first test, $10.0 \mathrm{~mL} 0.10 \mathrm{M}$ BTMAP-Vi in $2.0 \mathrm{M} \mathrm{KCl}$ (supporting electrolyte) was used as the negative electrolyte, and $5.0 \mathrm{~mL} 0.10 \mathrm{M}$ (TPABPy) $\mathrm{Cl}_{3}$ in $2.0 \mathrm{M} \mathrm{KCl}$ as the positive electrolyte. For the second test, $8.0 \mathrm{~mL} 1.20 \mathrm{M}$ BTMAP-Vi and $4.0 \mathrm{~mL} 1.50 \mathrm{M}$ (TPABPy) $\mathrm{Cl}_{3}$ were used without supporting electrolyte.

2.7. Density Functional Theory (DFT) Calculation. Gaussian 09 program was used to carry out all DFT calculations [10]. Initial configurations including GD3BJ (empirical dispersion correction) [11, 12] and SMD (implicit solvation model based on density) were optimized at the B3LYP/6-31G* level $[13,14]$. For each optimized structures, their HOMO/LUMO
TABLE 1: Physicochemical properties of TEMPO, TMAP-TEMPO, and (TPABPy) $\mathrm{Cl}_{3}$.

\begin{tabular}{lcccc}
\hline Electrolyte & $\begin{array}{c}\boldsymbol{E}_{1 / 2}(\mathrm{~V}, \mathrm{vs} . \\
\text { SHE })\end{array}$ & $k_{0}\left(\mathrm{cms}^{-1}\right)$ & $\boldsymbol{D}\left(\mathrm{cm}^{2} \mathrm{~s}^{-1}\right)$ & $P\left(\mathrm{~cm}^{2} \mathrm{~s}^{-1}\right)$ \\
\hline TEMPO & +0.745 & $6.62 \times 10^{-3}$ & $5.36 \times 10^{-6}$ & $\mathrm{NA}$ \\
TMAP- & +0.810 & $1.02 \times 10^{-2}$ & $3.48 \times 10^{-6}$ & $6.4 \times 10^{-10}$ \\
TEMPO & & & & \\
(TPABPy $\mathrm{Cl}_{3}$ & +0.967 & $7.50 \times 10^{-2}$ & $2.97 \times 10^{-6}$ & $1.8 \times 10^{-11}$ \\
\hline
\end{tabular}

NA means no data available.

orbitals were analyzed by Multiwfn [15], and the figures were drawn by VMD [16] package (with isosurface $=0.03$ a.u.); their Gibbs free energy values were calculated at the M062X/def2-SV [17, 18] level.

\section{Results and Discussion}

3.1. Structure and Composition of TEMPO. As illustrated in Scheme 2, (TPABPy) $\mathrm{Cl}_{3}$ is synthesized in a facile protocol, which includes the Zincke reaction [19], an $\mathrm{S}_{\mathrm{N}} 2$ reaction and ion exchange step. And the purification of each step can be performed by filtration without use of 


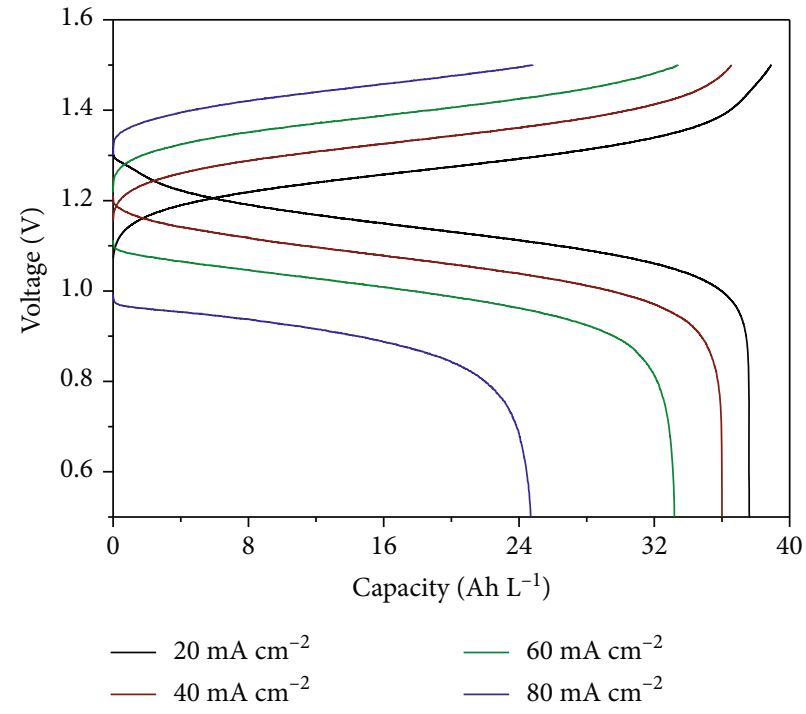

(a)

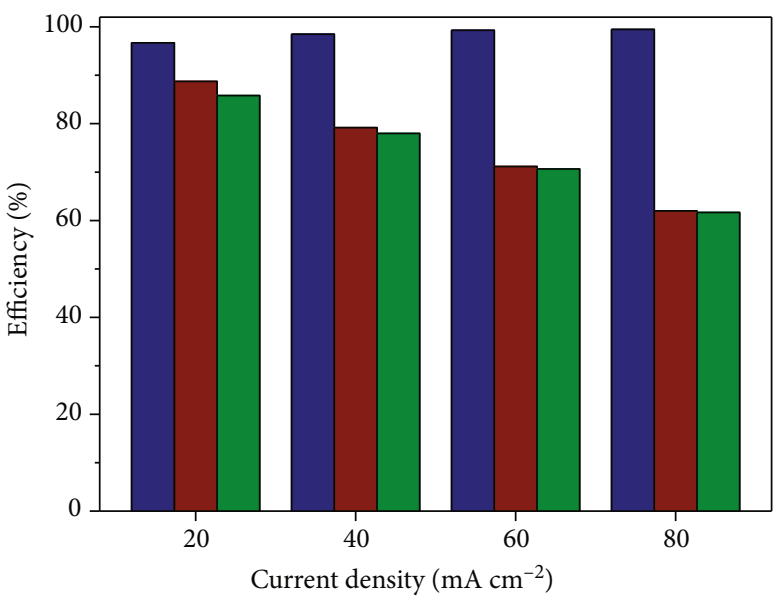

$\square \mathrm{CE}$

$\square \mathrm{VE}$

$\square \mathrm{EE}$

(b)

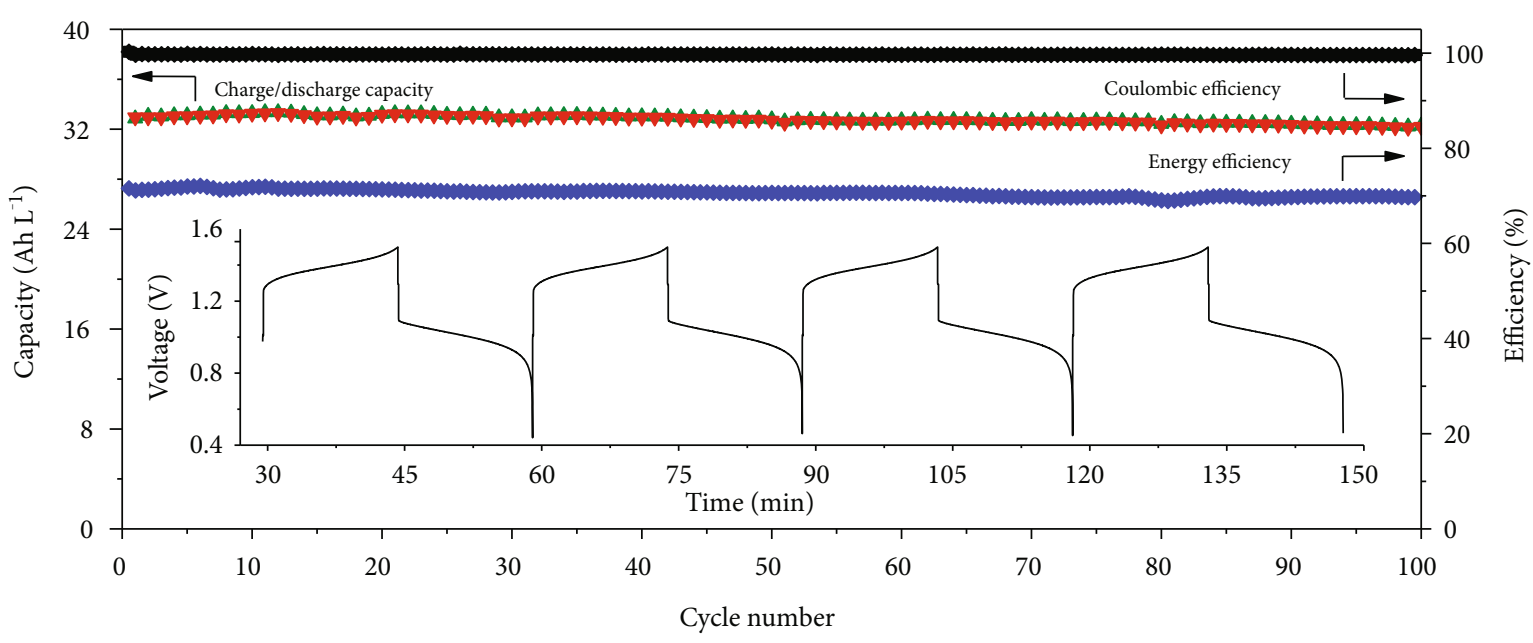

(c)

FIGURE 4: (a) Charge and discharge curves at various current densities with 1.5 and $0.5 \mathrm{~V}$ cut offs; (b) plot of average CE, VE, and EE at different current densities; (c) cycling performance of the ARFB at $60 \mathrm{~mA} \mathrm{~cm}^{-2}$, inset: the charge and discharge curves.

chromatography. The structure and composition of the product are investigated by ${ }^{1} \mathrm{H}-\mathrm{NMR}$ (Figures S1-S2) and single crystal structure analysis (Figure S3, Table S1).

The electronic effect of viologen on TEMPO is investigated by ${ }^{1} \mathrm{H}$ NMR (Figure 1(a), Figure S4). Figure 1(a) reveals a remarkable chemical shift to low field for the protons of methyl groups in the TEMPO ring, which illustrates an electron-withdrawing effect of viologen. This singlet peak splits into two singlet ones due to the nuclear overhauser effect (NOE, Figure S4). X-ray single crystallographic analysis confirms that $\mathrm{H}-\mathrm{H}$ distance between the protons at the 4-postion and a- $\mathrm{CH}_{3}$ in the TEMPO ring lies within internuclear distances for NOE $(<5 \AA)$ (see the red circle in Figure 1(b)).

The energy level of the lowest unoccupied molecular orbital (LUMO) and the highest occupied molecular orbital (HOMO) of the oxidized state is calculated at the B3LYP/6-
$31 \mathrm{G} *$ level. Figure 2 shows that the LUMO of (TPABPy $)^{4+}$ $(-4.572 \mathrm{eV})$ is lower than that of $(\text { TEMPO })^{+}(-4.293 \mathrm{eV})$, indicating that (TPABPy $)^{4+}$ has a higher electron affinity than does (TEMPO) ${ }^{+}$.

3.2. Electrochemical Performance. The redox potential is evaluated by cyclic voltammetry $(\mathrm{CV})$. Figure 3 reveals that the formal potential of (TPABPy) $\mathrm{Cl}_{3}(+0.967 \mathrm{~V}$, vs. SHE) is $0.222 \mathrm{~V}$ higher than that of TEMPO $(+0.745 \mathrm{~V}$, vs. SHE). The elevation of the redox potential can be attributed to the remarkable electron-withdrawing effect of the viologen unit, as evidenced by both NMR and the energy level analysis (vide supra). Both the diffusion coefficient and the rate constant are derived from linear sweep voltammetry (LSV, Figures S5-S8). The diffusion coefficient of (TPABPy) $\mathrm{Cl}_{3}$ is $2.97 \times 10^{-6} \mathrm{~cm}^{2} \mathrm{~s}^{-1}$, which is slightly lower than that of TEMPO (see Table 1). The decrease is understandable 


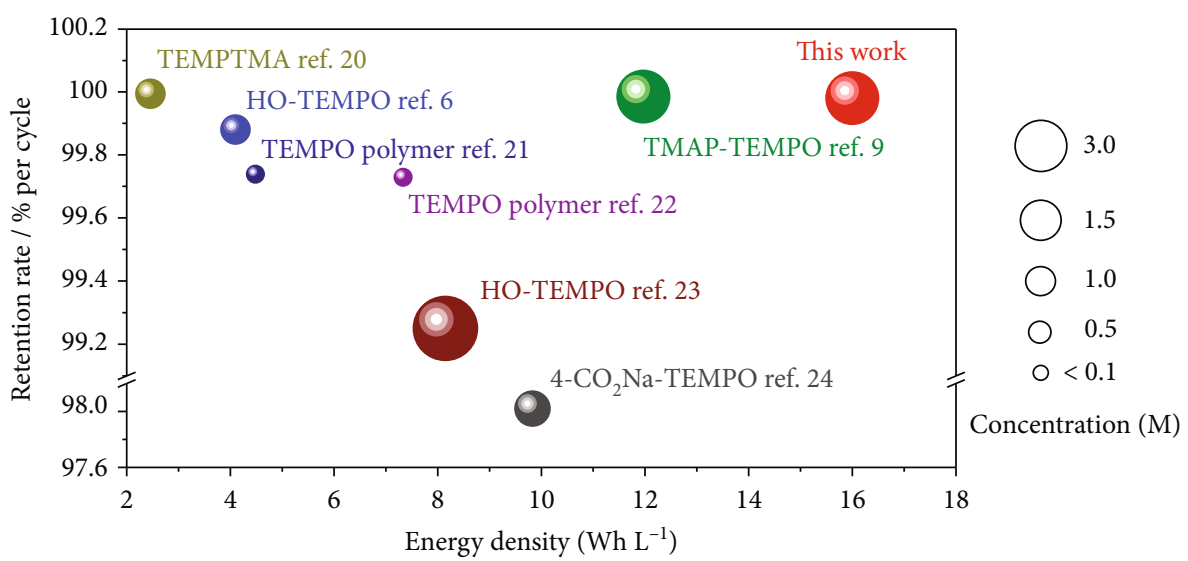

FIGURE 5: Comparison of capacity retention rate and energy density of the reported viologen(-)/TEMPO(+) ARFBs.

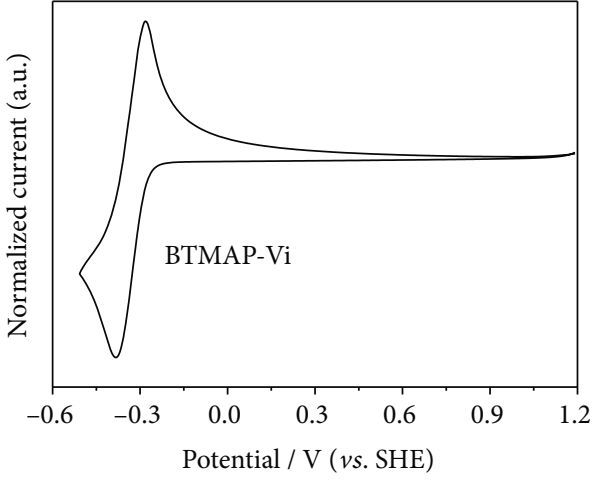

(a)

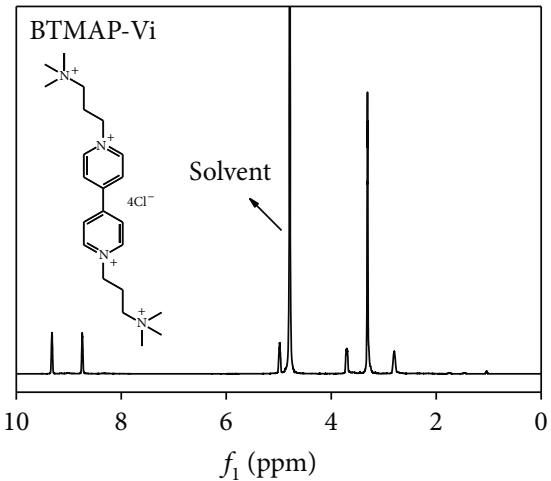

(b)

Figure 6: (a) CV and (b) ${ }^{1} \mathrm{H}$ NMR of the negative electrolyte after cycling test.

considering the higher molecular weight after decorating viologen. The rate constant of the one-electron oxidation reaction of (TPABPy)Cl $\mathrm{Cl}_{3}$ is $7.50 \times 10^{-2} \mathrm{~cm} \mathrm{~s}^{-1}$, which is higher than that of TEMPO $\left(6.62 \times 10^{-3} \mathrm{~cm} \mathrm{~s}^{-1}\right)$ and TMAP-TEMPO [9] $\left(1.02 \times 10^{-2} \mathrm{~cm} \mathrm{~s}^{-1}\right)$. This result illustrates that the viologen unit favors the electron transfer of TEMPO during the redox reaction. The above features of the (TPABPy $\mathrm{Cl}_{3}$ make it as a promising positive electrolyte for ARFBs.

3.3. Flow Battery Performance. As demonstrated, a battery is operated with $1.5 \mathrm{M}$ (TPABPy) $\mathrm{Cl}_{3}$ as the positive electrolyte and 1.2 M BTMAP-Vi as the negative electrolyte. Notably, no external supporting electrolyte is added in either side due to their high conductivity. Figure 3 reveals that the standard voltage of this battery is up to $1.30 \mathrm{~V}$. Figure 4(a) displays the rate performance with operating current from 20 to $80 \mathrm{~mA} \mathrm{~cm}^{-2}$. It is seen that the battery yields a high capacity of $37.6 \mathrm{Ah} \mathrm{L}^{-1}$ at $20 \mathrm{~mA} \mathrm{~cm}^{-2}$, and the capacity shows a mild decrease with increasing the current density. Figure 4(b) shows that the battery delivers a nearly $100 \%$ coulombic efficiency (CE) at each current density. The energy efficiency (EE) and voltage efficiency (VE) are found to be sufficiently high, e.g., $70.6 \% \mathrm{EE}$ and $71.1 \% \mathrm{VE}$ achieved at $60 \mathrm{~mA} \mathrm{~cm}^{-2}$. The high efficiency can be attributed to the high electrochemical reversibility of the two redox couples. Figure 4(c) shows the 100 -cycle test result at $60 \mathrm{~mA} \mathrm{~cm}^{-2}$ to evaluate the stability. This battery provides a high capacity retention of $99.98 \%$ per cycle, demonstrating its superior stability. It is noted that this battery represents the most stable one in the reported viologen(-)/TEMPO(+) ARFBs without using any additional supporting electrolyte (Figure 5) [6, 9, 20-24]. Postcell analysis is conducted by using $\mathrm{CV}$ and ${ }^{1} \mathrm{H}$ NMR to investigate the superior stability. As shown in Figure 6(a), the redox signal of (TPABPy) $\mathrm{Cl}_{3}$ is not seen in the negative electrolyte, suggesting the crossover of (TPABPy) $\mathrm{Cl}_{3}$ is negligible. In addition, Figure 6(b) only shows the proton resonance signals of the BTMAP-Vi. These results strongly verify the low crossover of the electroactive species in both sides, which contributes to the cycling stability of the battery.

\section{Conclusions}

In this work, viologen-decorated TEMPO ((TPABPy) $\left.\mathrm{Cl}_{3}\right)$ is developed as the positive electrolyte in neutral aqueous organic redox flow battery. The introduction of viologen unit is proved effective in increasing the TEMPO's aqueous solubility, elevating the redox potential and preventing its crossover. As a result, by pairing BTMAP-Vi as the negative electrolyte, an assembled flow battery using $1.5 \mathrm{M}$ (TPAB$\mathrm{Py}) \mathrm{Cl}_{3}$ in the positive side without any supporting electrolyte affords a high energy density of $19.0 \mathrm{Wh} \mathrm{L}^{-1}$ and a stable 
cycling performance with capacity retention of $99.98 \%$ per cycle. This work highlights the rational design of positive electrolyte for neutral ARFB, which will pave the road to develop energy-dense, stable, and lost-cost ARFBs.

\section{Data Availability}

The data that support the findings of this study are available from the corresponding author upon reasonable request.

\section{Conflicts of Interest}

The authors declare no conflict of interest.

\section{Authors' Contributions}

Shuzhi Hu and Liwen Wang contributed equally to this work.

\section{Acknowledgments}

This work was financially supported by the National Natural Science Foundation of China (21975081 and 21905114).

\section{Supplementary Materials}

NMR spectra of (TPPy)Cl and (TPABPy) $\mathrm{Cl}_{3}$, single crystal structure of (TPABPy) $\left(\mathrm{PF}_{6}\right)_{3}$, LSV curves of (TPABPy) $\mathrm{Cl}_{3}$, performance of the $0.10 \mathrm{M}$ BTMAP-Vi(-)/(TPABPy) $\mathrm{Cl}_{3}(+)$ battery, and aqueous solubility test of (TPABPy) $\mathrm{Cl}_{3}$. (Supplementary Materials)

\section{References}

[1] Y. X. Yao, J. F. Lei, Y. Shi, F. Ai, and Y. C. Lu, "Assessment methods and performance metrics for redox flow batteries," Nature Energy, vol. 6, no. 6, pp. 582-588, 2021.

[2] Z. J. Li and Y. C. Lu, "Material design of aqueous redox flow batteries: fundamental challenges and mitigation strategies," Advanced Materials, vol. 32, no. 47, article 2002132, 2020.

[3] R. Z. Feng, X. Zhang, V. Murugesan et al., "Reversible ketone hydrogenation and dehydrogenation for aqueous organic redox flow batteries," Science, vol. 372, no. 6544, pp. 836840, 2021.

[4] J. Cao, J. Tian, J. Xu, and Y. Wang, "Organic flow batteries: recent progress and perspectives," Energy \& Fuels, vol. 34, no. 11, pp. 13384-13411, 2020.

[5] Z. Li, S. Li, S. Q. Liu et al., "Electrochemical properties of an allorganic redox flow battery using 2,2,6,6-Tetramethyl-1-Piperidinyloxy and N-methylphthalimide," Electrochemical and Solid-State Letters, vol. 14, no. 12, pp. A171-A173, 2011.

[6] T. B. Liu, X. L. Wei, Z. M. Nie, V. Sprenkle, and W. Wang, "A total organic aqueous redox flow battery employing a low cost and sustainable methyl viologen anolyte and 4-HO-TEMPO catholyte," Advanced Energy Materials, vol. 6, no. 3, article 1501449, 2016.

[7] T. Janoschka, N. Martin, M. D. Hager, and U. S. Schubert, “An aqueous redox-flow battery with high capacity and power: the TEMPTMA/MV system," Angewandte Chemie, vol. 55, no. 46, pp. 14427-14430, 2016.
[8] Y. H. Liu, Q. R. Chen, P. Sun, Y. Li, Z. Yang, and T. Xu, "Organic electrolytes for aqueous organic flow batteries," Materials Today Energy, vol. 20, article 100634, 2021.

[9] Y. H. Liu, M. Goulet, L. C. Tong et al., "A Long-Lifetime allorganic aqueous flow battery utilizing TMAP-TEMPO radical," Chem, vol. 5, no. 7, pp. 1861-1870, 2019.

[10] M. J. Frisch, G. W. Trucks, H. B. Schlegel et al., Gaussian 09, Revision A.02, Gaussian, Inc., Wallingford CT, 2016.

[11] S. Grimme, S. Ehrlich, and L. Goerigk, "Effect of the damping function in dispersion corrected density functional theory," Journal of Computational Chemistry, vol. 32, no. 7, pp. 14561465, 2011.

[12] S. Grimme, "Accurate description of van der Waals complexes by density functional theory including empirical corrections," Journal of Computational Chemistry, vol. 25, no. 12, pp. 1463-1473, 2004.

[13] P. J. Stephens, F. J. Devlin, C. F. Chabalowski, and M. J. Frisch, "Ab initio calculation of vibrational absorption and circular dichroism spectra using density functional force fields," The Journal of Physical Chemistry, vol. 98, no. 45, pp. 1162311627, 1994.

[14] R. Ditchfield, W. J. Hehre, and J. A. Pople, "Self-consistent Molecular-Orbital methods. IX. An Extended Gaussiantype basis for molecular-orbital studies of organic molecules," The Journal of Chemical Physics, vol. 54, no. 2, pp. 724-728, 1971.

[15] T. Lu and F. Chen, "Multiwfn: a multifunctional wavefunction analyzer," Journal of Computational Chemistry, vol. 33, no. 5, pp. 580-592, 2012.

[16] W. Humphrey, A. Dalke, and K. Schulten, "VMD: visual molecular dynamics," Journal of Molecular Graphics, vol. 14, no. 1, pp. 33-38, 1996.

[17] Y. Zhao and D. G. Truhlar, "The M06 suite of density functionals for main group thermochemistry, thermochemical kinetics, noncovalent interactions, excited states, and transition elements: two new functionals and systematic testing of four M06-class functionals and 12 other functionals," Theoretical Chemistry Accounts, vol. 120, no. 1-3, pp. 215-241, 2008.

[18] F. Weigend and R. Ahlrichs, "Balanced basis sets of split valence, triple zeta valence and quadruple zeta valence quality for $\mathrm{H}$ to $\mathrm{Rn}$ : design and assessment of accuracy," Physical Chemistry Chemical Physics, vol. 7, no. 18, pp. 3297-3305, 2005.

[19] M. Eda, M. J. Kurth, and M. H. Nantz, "The solid-phase Zincke reaction: preparation of $\omega$-hydroxy pyridinium salts in the search for CFTR activation," The Journal of Organic Chemistry, vol. 65, no. 17, pp. 5131-5135, 2000.

[20] B. Hu, Y. J. Tang, J. Luo, G. Grove, Y. Guo, and T. L. Liu, "Improved radical stability of viologen anolytes in aqueous organic redox flow batteries," Chemical Communications, vol. 54, no. 50, pp. 6871-6874, 2018.

[21] T. Janoschka, N. Martin, U. Martin et al., “An aqueous, polymer-based redox-flow battery using non-corrosive, safe, and low-cost materials," Nature, vol. 527, no. 7576, pp. 7881, 2015.

[22] T. Hagemann, M. Strumpf, E. Schröter et al., “(2,2,6,6-Tetramethylpiperidin-1-yl)oxyl-Containing zwitterionic polymer as catholyte species for high-capacity aqueous polymer redox flow batteries," Chemistry of Materials, vol. 31, no. 19, pp. 7987-7999, 2019. 
[23] Z. F. Huang, C. W. M. Kay, B. Kuttich et al., "An "interactionmediating" strategy towards enhanced solubility and redox properties of organics for aqueous flow batteries," Nano Energy, vol. 69, article 104464, 2020.

[24] B. Liu, C. W. Tang, H. R. Jiang, G. Jia, and T. Zhao, "Carboxylfunctionalized TEMPO catholyte enabling High-CyclingStability and high-energy-density aqueous organic redox flow batteries," ACS Sustainable Chemistry \& Engineering, vol. 9, no. 18, pp. 6258-6265, 2021. 